\title{
Adaptive Control of Hybrid Battery Energy Storage Systems under Capacity Fade
}

\author{
Nilanjan Mukherjee, Dani Strickland, Mina Abedi Varnosfaderani \\ ASTON UNIVERSITY \\ Power Engineering and Power Electronics Group \\ Birmingham, United Kingdom \\ Tel.: +44 / (0) - 121204 3615, 7891806767
}

E-Mail: mukhern2@ aston.ac.uk, D.STRICKLAND@aston.ac.uk, abedivam@aston.ac.uk

URL: http://www.aston.ac.uk/eas/research/groups/pepe/

\section{Acknowledgements}

Authors would like to acknowledge Engineering and Physical Science Research Council (EPSRC grant no. EP/1008764/1), UK for providing financial supports for this research project.

\section{Keywords}

«Batteries», «Converter Control», «Energy Storage», «Hybrid power integration», «Adaptive Control»

\begin{abstract}
There is an increasing call for applications which use a mixture of batteries. These hybrid battery solutions may contain different battery types for example; using second life ex-transportation batteries in grid support applications or a combination of high power, low energy and low power, high energy batteries to meet multiple energy requirements or even the same battery types but under different states of health for example, being able to hot swap out a battery when it has failed in an application without changing all the batteries and ending up with batteries with different performances, capacities and impedances. These types of applications typically use multi-modular converters to allow hot swapping to take place without affecting the overall performance of the system. A key element of the control is how the different battery performance characteristics may be taken into account and the how the power is then shared among the different batteries in line with their performance. This paper proposes a control strategy which allows the power in the batteries to be effectively distributed even under capacity fade conditions using adaptive power sharing strategy. This strategy is then validated against a system of three different battery types connected to a multi-modular converter both with and without capacity fade mechanisms in place.
\end{abstract}

\section{Introduction}

New batteries can be a costly investment in an energy storage applications, therefore to assist with cost reductions, there is a significant interest in industry in using a second life transportation battery based energy storage solution to meet grid frequency response rates, power and energy requirements under different applications [1]. The idea behind these applications is to use the batteries all the way through until end of life to get the maximum benefit out of the initial investment. With the lack of development of a second life supply chain, these batteries may have different battery chemistries (e.g. lead acid, nickel metal hydride (NiMH), lithium-ion) and come in a range of sizes (both in terms of power and energy) depending on the application (e.g. EV, HEV, bus, and motorbike). Therefore, each battery within a hybrid solution may be considered to have different capacity, nominal voltage, initial state-ofcharge (SOC), charging/discharging limits, physical size. These batteries will tend to perform differently and degrade differently. This paper presents a method of dealing with distributing the power among the batteries even under a rapid capacity fade situation. This paper starts by describing a suitable dc-side modular converter which can integrate these hybrid batteries into a grid-tie converter. Thereafter, the paper reports an adaptive distributed power sharing strategy of hybrid batteries which distributes the required grid side power among the battery modules according to their characteristics. 
An online parameter estimation method has also been discussed which can track the variation of battery capacity and internal impedance. Finally experimental validations have been presented to demonstrate the effectiveness of the proposed strategy under a capacity fade condition using a hybrid battery energy storage system prototype.

\section{Multi-modular Converter}

Past researches on battery energy storage system (BESS) have been done using a single type of batteries where a single-stage or a two-stage converter along with an appropriate cell balancing strategy was employed [2] - [3]. Modular power converter has been employed in BESS but at a high power levels and/or in medium voltage grid connection as discussed in [4] - [6]. Predominantly, a cascaded H-bridge type converter was used in those researches reported in [4] - [6] because of overall modularity, scalability, ease of control, multilevel waveform at the output. However, this work deals with a hybrid second life battery based energy storage system using different battery types where conventional two level converters are insufficient because of reliability issue [7]. A multi-modular converter with a battery per module represents a good way of dealing with a hybrid energy storage system as it allows for reasonable system reliability while enabling "hot swap" capability and ensuring independent control of each module battery. According to [7], a fault-tolerant cascaded dc-side modular converter topology is a suitable converter topology to use in this application as shown in Fig. 1. The switches $S_{i}, S_{i i}$ works in PWM mode to control the power flow while $T_{i}, T_{i i}$ can be used bypassing a faulty module ( $T_{i}$ is OFF and $T_{i i} \mathrm{ON}$ ). In this paper a boost module is used as a low voltage battery system is considered.

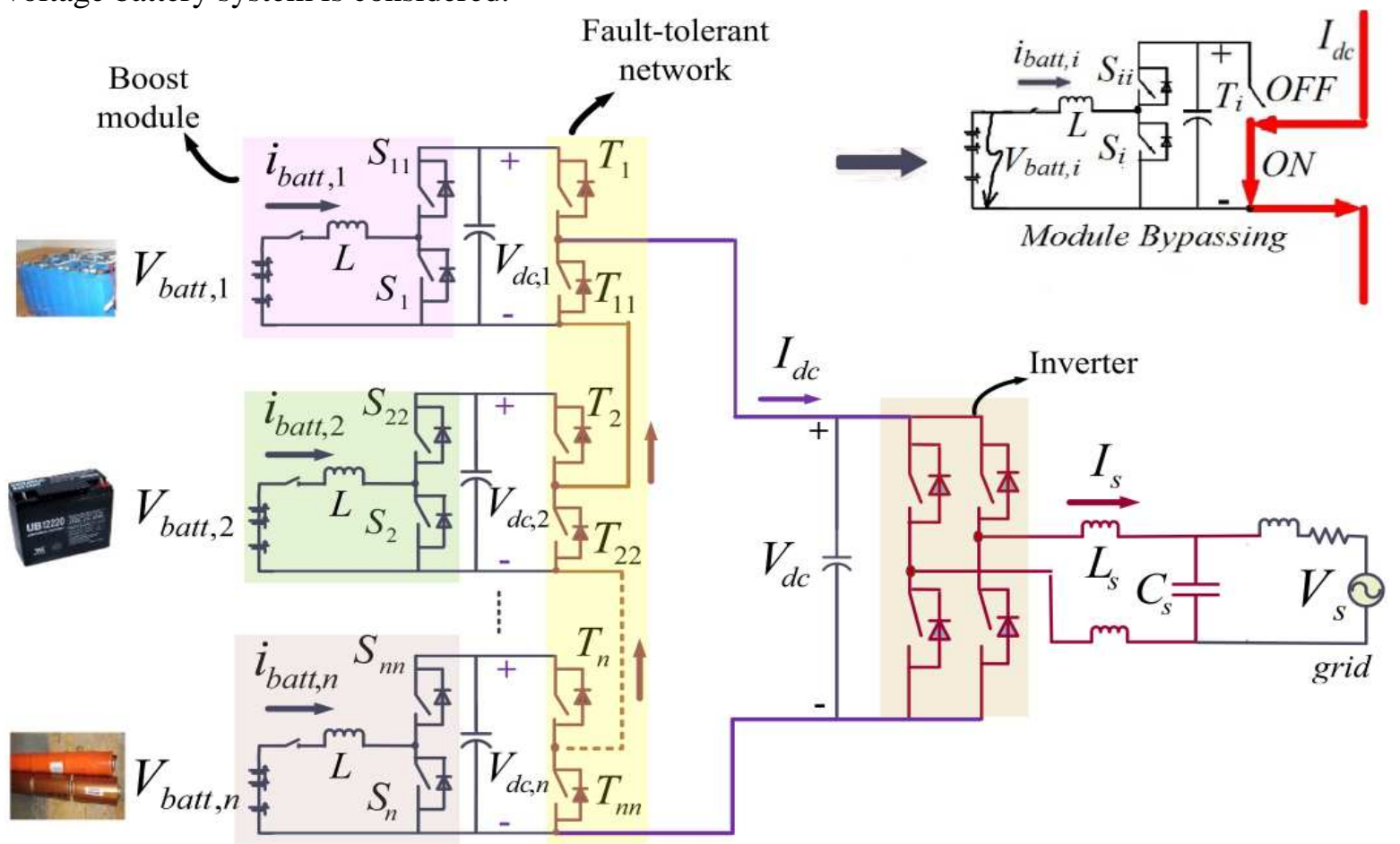

Fig. 1 Dc-side multi-modular converter structure for hybrid battery integration

\section{Adaptive Power Sharing of Hybrid Batteries}

There could be a hybrid mix of modules within the BESS in second life application. Therefore each of these module types will charge/discharge at different rates and have different maximum/minimum safe amounts of charge and voltages. Ideally none of the modules should be bypassed unless an abnormal condition or a fault is detected, so each battery module could take a proportionate share of the total power contribution relative to the state of each battery. Therefore, the charging/discharging strategy used in this paper is designed to ensure that the charging/discharging trajectory of the hybrid modules during a charging or discharging cycle will all arrive at their respective maximum and minimum state- 
of-charge (SOC) at the same time. The concept can be explained with the help of Fig. 2 which shows the estimated charge trajectory of three batteries with time.

Before developing a sharing strategy, the following terminology and assumptions are listed;

- A battery module is a collection of identical cells types

- A battery capacity has been taken as the maximum charge left ( $Q_{\max }$ in $\mathrm{C}$ or $\mathrm{Ah}$ ) that a battery can deliver to a load

- A module battery is modelled as an open circuit voltage $(O C V)$ with a series impedance $(Z)$.

Each battery and module contains a balancing network and it is assumed that within each sub modules the following parameters are equal and where necessary known from pre-characterization:

- Maximum and minimum voltage limits $\left(O C V_{\max }\right.$ or $\left.O C V_{\min }\right)$

- Maximum and minimum charge limits or Capacity $\left(Q_{\max }\right)$

- Internal impedance $(Z)$

- Relationship between SOC and OCV

Previous research shows battery SOC and capacity are related through a linear coulomb counting equation [8] where the SOC can be considered a percentage representation of charge.

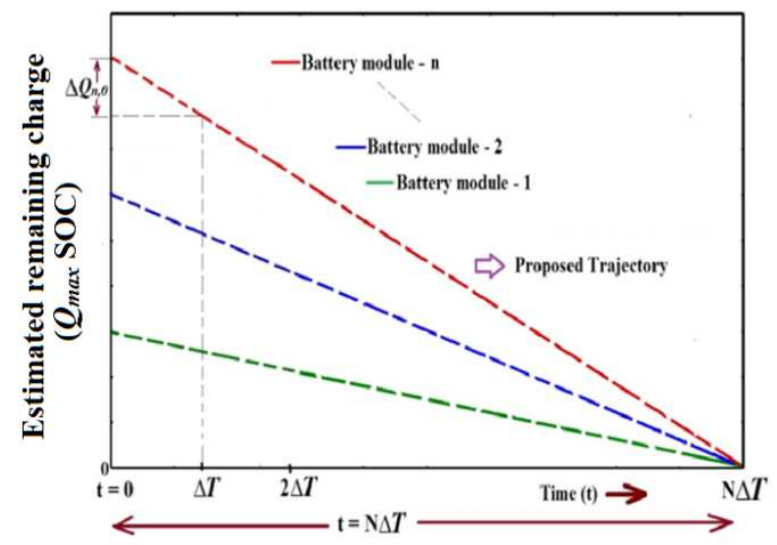

(a)

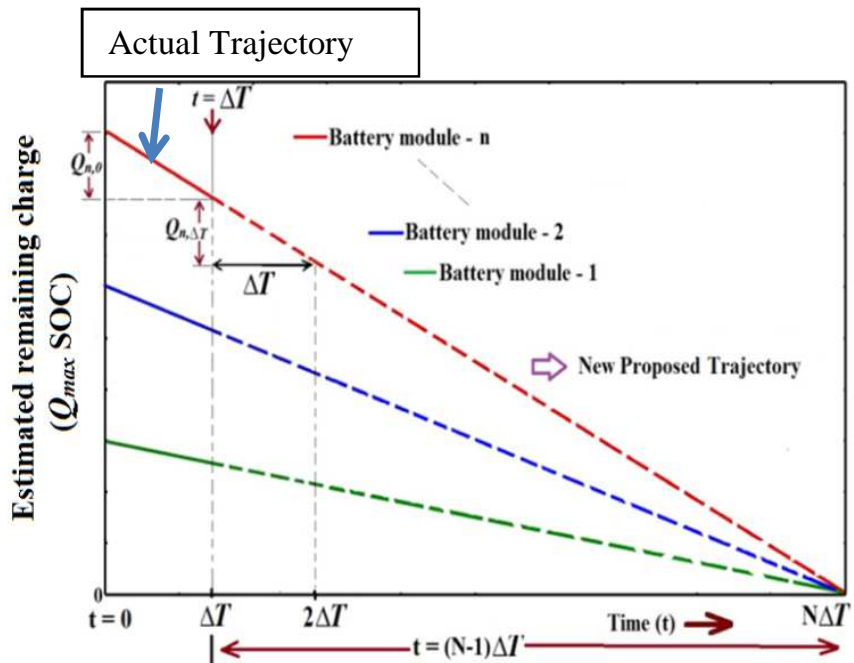

(b)

Fig. 2 Proposed trajectory to deal with hybrid batteries during discharging: a) at $\mathrm{t}=0, \mathrm{~b}$ ) at $\mathrm{t}=\Delta T$

\section{Weighting factor based strategy}

The total time to charge or discharge can be considered to be divided into a total number, $N$ of small equal time periods $(\Delta T)$ dependent on the sample time, where the required change in charge of each module at the $k^{\text {th }}$ sample instant is $\Delta Q_{n(k \Delta T)}$ as shown in Fig. 2.

Therefore, $\Delta Q_{n(k \Delta T)}$, at the $k^{\text {th }}$ sample time can be written as:

$\Delta Q_{n(k \Delta T)}=\frac{Q_{\max , n} S O C_{n(k \Delta T)}}{N}$

Where $Q_{\max , n} S O C_{n(k \Delta T)}$ represents the total remaining charge in module $n$ at the $k^{\text {th }}$ sample instant and $N$ is the total number of sample periods before the battery is fully discharged.

A similar expression can be derived for charging: 
$\Delta Q_{n(k \Delta T)}=\frac{Q_{\max , n}\left(1-S O C_{n(k \Delta T)}\right)}{N}$

Charging/discharging depends on the module current. Previous research shows battery SOC and capacity are related through a linear coulomb counting equation [8]. The fundamental charge equation, the relation between charge and SOC for a module is given by (3) where the $Q_{0}$ is the initial charged stored.

$Q(t)=Q_{0}+\int_{0}^{T} i_{\text {batt }} d t$

Over the small sample period, $\Delta T$, (3) can be written as

$\int_{(k-1) \Delta T}^{k \Delta T} i_{b a t t} d t=\Delta Q_{n(k \Delta T)}$

Within the small time period $\Delta T$, it is reasonable to assume the current is constant. Substituting from (1) into (4) for a single module $n$, with a small discharging current gives:

$I_{b a t t, n,(k)}=\frac{\Delta Q_{n(k \Delta T)}}{\Delta T}=\frac{Q_{\max , n} S O C_{n(k \Delta T)}}{N \Delta T}$

A similar expression can be derived for charging from (2) for the module $n$,

$I_{b a t t, n(k)}=\frac{Q_{\max , n}\left(1-S O C_{n(k \Delta T)}\right)}{N \Delta T}$

Due to the fact that we require the total charge or discharge time to be the same between the modules $(N \Delta T)$, it can be seen that the current needs to be split in proportion to the remaining charge $\left(Q_{\max } S O C\right)$ available. The current should be adjusted at each time step to ensure the trajectory remains constant over the time period and changes in battery condition are accounted for.

To calculate the desired current for a given total power, the converter power balance equation over each sample period, can be used as shown below.

$P=\sum_{m=1}^{n} V_{\text {batt }, m,(k)} I_{\text {batt }, m,(k)}$

Where $n$ is the number of active modules and each module, $m$ has a different battery terminal voltage, $V_{\text {batt }, m}$ and current $I_{\text {batt }, m}$.

Now, substituting (5) into (7) for each module $m$ discharging, gives:

$\mathrm{P}=\sum_{\mathrm{m}=1}^{\mathrm{n}} V_{\text {batt }, m,(k)} \frac{Q_{\max , m} S O C_{m(k \Delta T)}}{N \Delta T}$

To find, say, $I_{b a t t, 1,(k)}$ and eliminate $N \Delta T$ from (8) which is equal for all modules, substitute for $N \Delta T$ from (5):

$P=\sum_{\mathrm{m}=1}^{\mathrm{n}} V_{\text {batt }, m,(k)} \frac{Q_{\max , m} S O C_{m(k \Delta T)}}{\left(\frac{\left(Q_{\max , 1} S O C_{1(k \Delta T)}\right)}{I_{\text {batt }, 1,(k)}}\right)}$

Re-arranging gives:

$I_{b a t t, 1,(k)}=\mathrm{P}\left(\frac{Q_{\max , 1} S O C_{1(k \Delta T)}}{\sum_{m=1}^{n} V_{b a t t, m,(k)} Q_{\max , m} S O C_{m(k \Delta T)}}\right)$

The desired current for the $i^{\text {th }}$ module will therefore be:

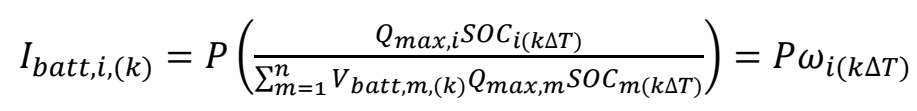


A similar expression for charging can be derived:

$I_{b a t t, i(k)}=\mathrm{P}\left(\frac{Q_{\max , i}\left(1-S O C_{i(k \Delta T)}\right)}{\sum_{m=1}^{n} V_{b a t t, m, k)} Q_{m a x}{ }_{m} S O C_{m(k \Delta T)}}\right)=P \omega_{i(k \Delta T)}$

The $\omega_{i}$ is the weighting function for the $i^{\text {th }}$ module. This weighting factor is different during charging and discharging. Where, the module battery $S O C_{n(k \Delta T)}$ is a function of open-circuit voltage $O C V_{i(k \Delta T)}$ at the sample time and $\omega_{i(k \Delta T)}$ is the weighting function over a sample period $k \Delta T$ and depends on $S O C$ and $Q_{\max }$ sample values.

\section{Online battery parameter tracking}

In this hybrid battery application, it is important that the battery capacity (or $Q_{\max }$ ) and internal impedance $(Z)$ are tracked during the battery operation because the long term battery degradation and/or temperature variation will consequently affect this capacity [8]. For example, capacity degrades it may be necessary for that battery to draw a lower current share in discharging. The initial capacity and impedance can be found through pre-characterization. However, it is necessary to track the $Q_{\max , i}$ and $Z$ to assist with the power sharing and to monitor $\mathrm{SOH}$ online during the converter operation.

Impedance Estimation: Impedance is a very important parameter within the battery model and can also act as an indication of state-of-health ( $\mathrm{SOH})$ of a battery and provides failure status [9] - [10]. This paper uses a high frequency converter ripple based online impedance estimation method. The concept is to use the high frequency inductor ripple current of the associated dc-dc converter and corresponding high frequency ripple of the battery terminal voltage to calculate the internal impedance. The switching frequency is 10 's of $\mathrm{kHz}$, so it can be assumed that the SOC does not change significantly during such small switching interval. Therefore, $O C V$ can also be assumed constant within that interval. Therefore, two different equations can be written within a switching cycle $T_{s}$, a) at $t=0$ and b) at $t=d T_{s}$ as shown in (13) and (14) with reference to Fig. 3. The internal impedance can be calculated using (15). The ripple components of battery current and voltage can be extracted from the measured current and voltage using a low pass filter with a cut-off frequency $1 / 10^{\text {th }}$ of the switching frequency. After extracting the ripple part of voltage and current, the magnitude impedance $(Z)$ can be calculated. The method has been validated using an EIS based method at ambient temperature as shown in Table I. The EIS method gives a separate real $(R)$ and imaginary $(X)$ component at $10 \mathrm{kHz}$ whereas the ripple based method provides the total $Z$. It can be seen that the total impedance $(Z)$ values are within $\pm 15 \%$ under charging and discharging conditions. The accuracy of this ripple based method is dependent on the accuracy with which the ripple can be measured. A more careful design could be improve this.

$$
\begin{aligned}
& V_{\text {batt }_{\text {min }}}=O C V \pm i_{\text {batt }_{\text {max }}} Z \text { at } t=0 \text { Or } \\
& V_{\text {batt }_{\text {max }}}=O C V \pm i_{\text {batt }_{\text {min }}} Z \text { at } t=0 \\
& V_{\text {batt }_{\text {min }}}=O C V \pm i_{\text {batt }_{\text {max }}} Z \text { at } t=d T_{s} \text { Or } \\
& V_{\text {batt }_{\text {max }}}=O C V \pm i_{\text {batt }_{\text {min }}} Z \text { at } t=d T_{s} \\
& Z=\left|\frac{\left(V_{\text {batt }_{\text {max }}}-V_{\text {batt } \left._{\text {min }}\right)}\right)}{\left(i_{\text {batt }_{\text {max }}}-i_{\text {batt }_{\text {min }}}\right)}\right|=\frac{\left|\left(\Delta V_{\text {batt }}\right)\right|}{\left|\left(\Delta i_{\text {batt }}\right)\right|}
\end{aligned}
$$




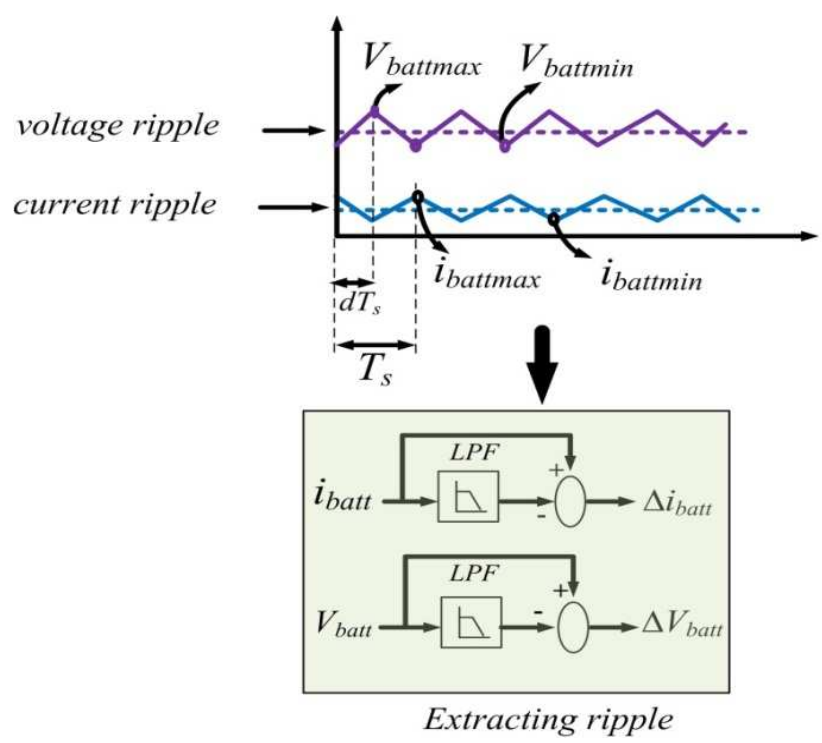

Fig. 3 Converter ripple based impedance estimation technique

Table I Comparison of ripple based impedance estimation method and EIS based method

\begin{tabular}{|l|l|l|l|l|l|l|l|l|}
\hline $\begin{array}{l}\text { Battery } \\
\text { types }\end{array}$ & $\begin{array}{l}\text { EIS } \\
\text { Discharge } \\
(R)\end{array}$ & $\begin{array}{l}\text { EIS } \\
\text { Discharge } \\
(X)\end{array}$ & $\begin{array}{l}\text { Total Z } \\
\text { EIS } \\
(\text { dischar } \\
\text { ge })\end{array}$ & $\begin{array}{l}\text { EIS } \\
\text { charge } \\
(R)\end{array}$ & $\begin{array}{l}\text { EIS } \\
\text { charge } \\
(X)\end{array}$ & $\begin{array}{l}\text { Total Z } \\
\text { EIS } \\
(\text { charge })\end{array}$ & $\begin{array}{l}\text { Ripple } \\
\text { based } \\
\text { discharge } \\
(Z)\end{array}$ & $\begin{array}{l}\text { Ripple } \\
\text { based } \\
\text { charge }(Z)\end{array}$ \\
\hline $\begin{array}{l}12 \mathrm{~V}, 10 \mathrm{Ah} \\
(\text { lead acid) }\end{array}$ & $23 \mathrm{~m} \Omega$ & $21 \mathrm{~m} \Omega$ & $31 \mathrm{~m} \Omega$ & $24 \mathrm{~m} \Omega$ & $22 \mathrm{~m} \Omega$ & $32 \mathrm{~m} \Omega$ & $28 \mathrm{~m} \Omega$ & $29 \mathrm{~m} \Omega$ \\
\hline $\begin{array}{l}7.2 \mathrm{~V}, \\
\begin{array}{l}6.5 \mathrm{Ah} \\
(\mathrm{NiMH})\end{array}\end{array}$ & $24 \mathrm{~m} \Omega$ & $30 \mathrm{~m} \Omega$ & $38 \mathrm{~m} \Omega$ & $23 \mathrm{~m} \Omega$ & $32 \mathrm{~m} \Omega$ & $40 \mathrm{~m} \Omega$ & $35 \mathrm{~m} \Omega$ & $33 \mathrm{~m} \Omega$ \\
\hline
\end{tabular}

Capacity Estimation: The method adopted in this paper to track the capacity is similar to [8] where the SOC against capacity coulomb counting equation was used to estimate the capacity when the SOC was known or obtained from a SOC-OCV look-up table. In this work, the same equation is used to estimate the capacity where the SOC is calculated from SOC-OCV relationship. The method is explained in steps:

i) Obtain the internal impedance $(Z)$ using the ripple based method as explained above.

ii) Find the corresponding OCV using the impedance based battery model, i.e. $O C V=V_{\text {batt }}+$ $i_{\text {batt }} Z$ when discharging and $O C V=V_{\text {batt }}-i_{\text {batt }} Z$ for charging.

iii) Find the corresponding SOC using SOC-OCV derived function or a look-up table

After obtaining SOC from the above, the capacity (or maximum charge left) can be found using (16)

$Q_{\max }=\left|\frac{\int i_{\text {batt }} d t}{\operatorname{SOC} C_{0} \sim \operatorname{SOC}(t)}\right|$

In order to assist with the power sharing, the initial capacity of a battery module has to be known as the capacity estimation process (expression (16)) is not instantaneous because the SOC and the integration of current are slow changing variables. In order to validate the method, a $12 \mathrm{~V} 10 \mathrm{Ah}$ lead acid battery was used. Fig. 4(a) is presented to show how the method tracks the capacity. The initial capacity at $t=0 \mathrm{~s}$ was assumed to be $8 \mathrm{Ah}$ and the estimation process was started at $t=1 \mathrm{~s}$. It can be seen that the method tracks the actual battery capacity over a couple of seconds. The time taken by the estimation process is dependent on the difference between the initial capacity and the actual capacity. 
The time to track the capacity could take several seconds to reach to the actual capacity. This time (to track the actual capacity) is negligible compared to overall charge or discharge time of a battery which is in the order of several minutes or hours.

In order to show tracking under degradation, Fig. 4(b) shows the capacity estimation process when two 10Ah batteries are put in parallel at a constant current. This means the effective charge/maximum capacity will be approximately $20 \mathrm{Ah}$ at start. Fig. 4(b) shows that the process is able to detect the battery capacity; when one of the battery modules was taken out to reduce the overall capacity or to emulate the capacity fade. The process is capable of tracking such variation with a accurately over a small time scale. The error in the estimation process is mainly due to the inaccuracy in the SOC estimation. This can be improved if more accurate estimation and measurement processes are employed.
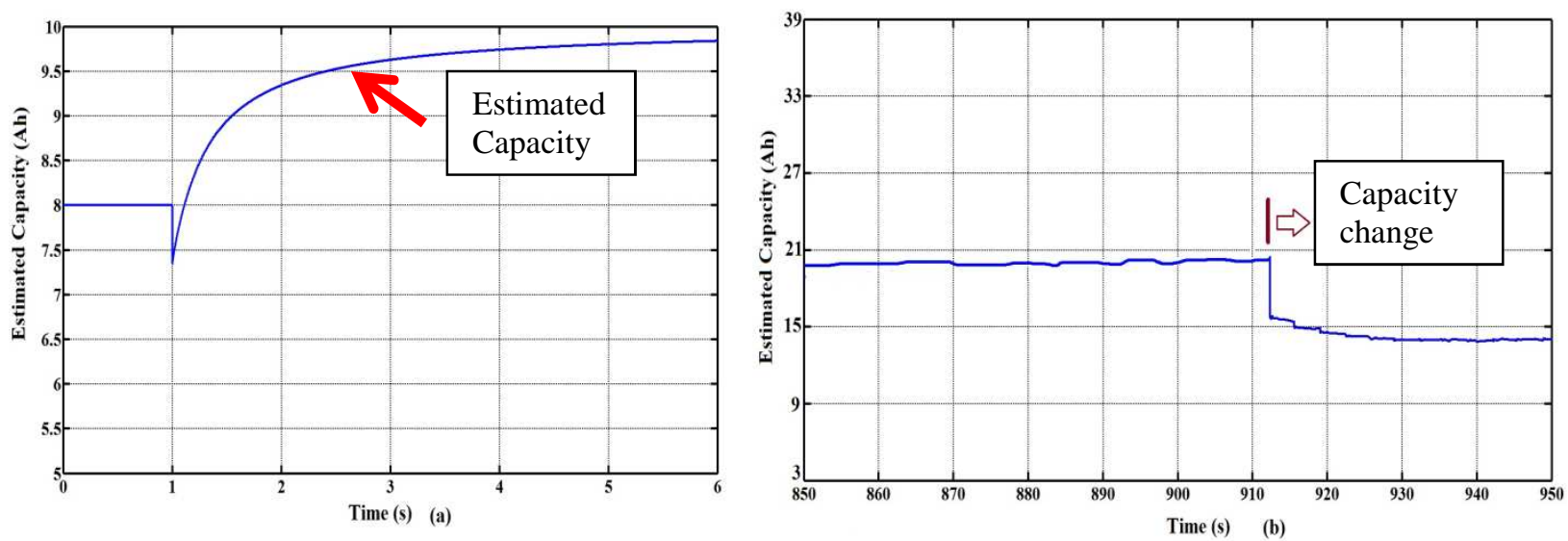

Fig. 4 Validation of capacity estimation process for two 12V, 10Ah lead acid batteries in parallel: a) capacity estimation for one $12 \mathrm{~V}, 10 \mathrm{Ah}$ battery, b) forced capacity fade condition

\section{Closed loop control structure}

The dynamic equations of an active module $(i)$ of the converter in Fig. 1 can be written in (17) - (18) assuming $T_{i}$ ON and $T_{i i} \mathrm{OFF}$ :

$$
\begin{aligned}
& L \frac{\mathbf{d} i_{\text {batt }, i}}{\mathbf{d} t}+R i_{\text {batt }, i}+\left(1-d_{i}\right) V_{d c, i}=V_{b a t t, i} \forall i=1 \ldots n \\
& C \frac{\mathbf{d} V_{d c, i}}{\mathbf{d} t}-\left(1-d_{i}\right) i_{b a t t, i}=-I_{d c} \forall i=1 \ldots n
\end{aligned}
$$

Total dc link voltage:

$V_{d c}=V_{d c, 1}+V_{d c, 2}+\cdots+V_{d c, n}$

The power balance equations of the system and the modules are given in (20) and (21) where $\eta_{i n v}$ is the inverter efficiency and $\eta_{i}$, is the efficiency of the dc-dc modules. In this condition, $V_{d c, i}$ becomes proportional to the module power $\left(V_{b a t, i} i_{b a t t, i}\right)$ because $I_{d c}$ is common across all the modules.

$V_{d c} I_{d c}=\eta_{i n v} V_{s} I_{s} \cong V_{s} I_{s}=P$

$V_{d c, i} I_{d c}=\eta_{i} V_{b a t t, i} i_{\text {batt }, i} \forall i=1 \ldots n$

The aims of the closed control are: a) to control the total dc-link voltage $\left(V_{d c}\right)$ to a constant value for to allow the line side inverter to operate according to the grid side power demand, b) controls each module capacitor voltage $\left(V_{d c, i}\right)$ to different voltage levels to independently control each of the modules. Therefore, the control system calculates the different module voltage references $\left(V_{d c, 1}{ }^{*}, V_{d c, 2}{ }^{*}\right.$ $\left.\ldots V_{d c, n}{ }^{*}\right)$ where the sum of all these references is a fixed central dc-bus reference $\left(V_{d c}{ }^{*}\right)$ for the inverter. These are derived using the power balance equations (21) and weighting factors and are 
shown in (22) to (23) assuming $\eta_{i} \approx 1$. A binary control status signal from switches $T_{i}, T_{i i}$ (from Fig. 1 ) is used in (23) to adjust the voltage reference if any of the modules are bypassed, as the control signals of the fault tolerant leg $T_{i}, T_{i i}$ are either fixed at 0 or 1 .

According to (11) or (12), $i_{\text {batt }, i}{ }^{*} \propto \omega_{i}$ and therefore:

$V_{d c, i}{ }^{*} I_{d c}=V_{b a t t, i} i_{b a t t, i}{ }^{*}$ or $V_{d c, i}{ }^{*} \propto V_{b a t t, i} \omega_{i} \forall i=1 \ldots n$ for a constant $I_{d c}$

$\sum V_{d c, i}{ }^{*}=V_{d c}{ }^{*}, V_{d c, i}{ }^{*}=V_{d c}{ }^{*} \frac{\omega_{i} V_{\text {bat } t, i} T_{i}}{\sum_{k=1}^{n} \omega_{k} V_{b a t t, k} \cdot T_{k}} \forall i=1 \ldots n$

Fig. 5 shows the proposed distributed voltage based control architecture. The line side control structure for grid support applications is presented in Fig. 6. The rotating frame based approach has been followed for the inverter where the ' $q$ ' axis is designed as an active power axis and ' $d$ ' axis the reactive power axis. The change-over of weighting factor between charging and discharging is performed using the sign of $i_{s q}{ }^{*}$ which is positive for discharging and negative for charging.

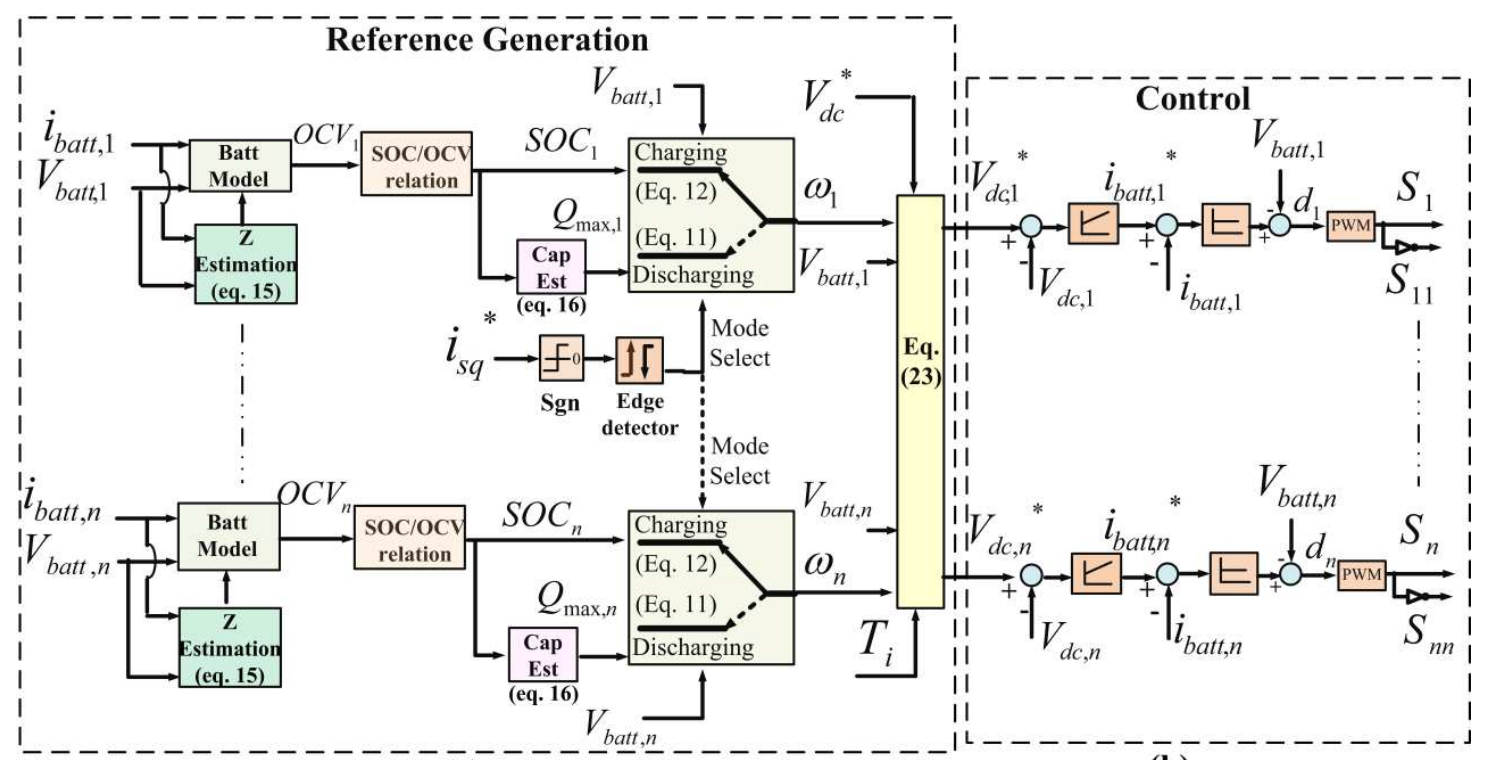

(a)

(b)

Fig. 5 Proposed closed loop control structure of the converter

\section{Experimental validation}

Three different batteries are used in the experimental validation as shown in Table II. All the converter modules have the same switches and passive components. The overall converter was tested in grid connected condition at a constant power level to prove the concept of adaptive power sharing. Fig. 7 shows the laboratory built scale-down prototype. The experimental results were presented in two ways: a) full charging and discharging trajectory under capacity fade and b) transient current sharing under those conditions. Fig. 8 shows the discharging trajectory using the distributed strategy under capacity fade. It can be seen that the current sharing changes when one of the battery module is dynamically taken out. This result shows that all the modules exhausting at the same time even under variation of battery capacity. Similar result has been presented in charging mode also in Fig. 9. It can be seen that the current in the battery module -1 reduces while the other module currents increase to compensate the power. The grid current remains unaffected under this operation. These results reveal that the proposed strategy is fully adaptive in nature and capable of detecting the capacity fade and dynamically change the power sharing to optimally use a set of hybrid batteries. 
Table II: Parameters for experimental implementation

\begin{tabular}{|l|l|}
\hline Test Power Rating & $500 \mathrm{~W}$ \\
\hline Grid voltage and frequency & Single phase $90 \mathrm{~V}(\mathrm{rms}), 50 \mathrm{~Hz}$ \\
\hline Operating central DC-link voltage & $150 \mathrm{~V}$ \\
\hline Semiconductor device/module & $100 \mathrm{~V} 40 \mathrm{~A}(\mathrm{FDPF} 085 \mathrm{~N} 10 \mathrm{~A}), R_{\text {dson }}=6 \mathrm{~m} \Omega$ \\
\hline Module inductors $(L)$ and capacitors $(C)$ & $1.5 \mathrm{mH}, 15 \mathrm{~A}$ and $2700 \mu \mathrm{F}, 200 \mathrm{~V}$ \\
\hline Battery module -1 (lead acid) & $12 \mathrm{~V}, 10 \mathrm{Ah}\left(O C V_{\max }=13.8 \mathrm{~V}, O C V_{\min }=9.6 \mathrm{~V}, \mathrm{Z}=0.024 \Omega\right)$ \\
\hline Battery module $-2($ lead acid $)$ & $24 \mathrm{~V}, 16 \mathrm{Ah}\left(O C V_{\max }=28 \mathrm{~V}, O C V_{\min }=18 \mathrm{~V}, \mathrm{Z}=0.024 \Omega\right)$ \\
\hline Battery module $-3(\mathrm{NiMH})$ & $7.2 \mathrm{~V}, 6.5 \mathrm{Ah}\left(O C V_{\max }=8.5 \mathrm{~V}, O C V_{\min }=5.5 \mathrm{~V}, \mathrm{Z}=0.023 \Omega\right)$ \\
\hline Switching frequency & $10 \mathrm{kHz}$ \\
\hline
\end{tabular}

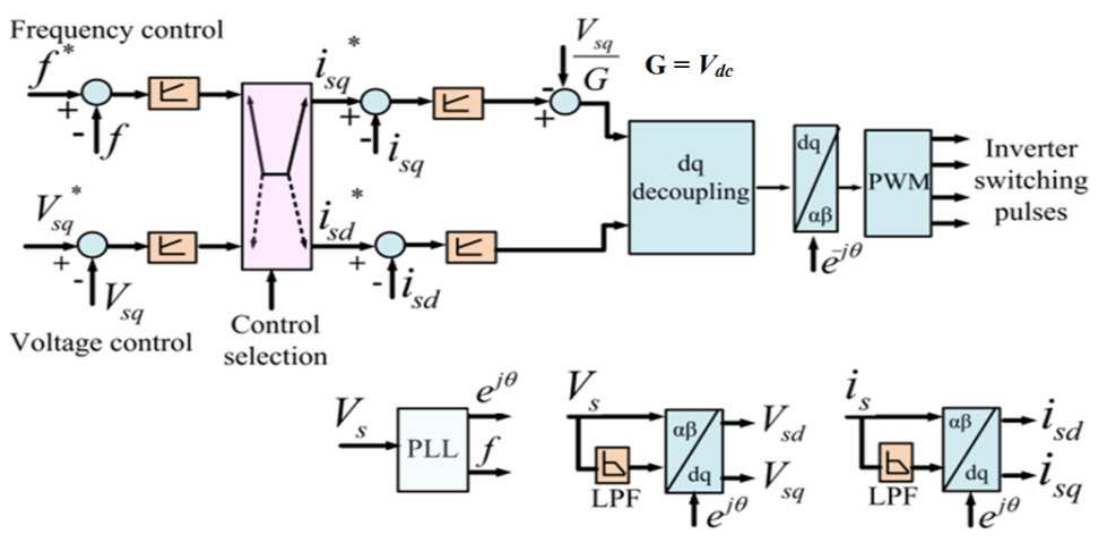

Fig. 6 Rotating frame based control for the line side inverter

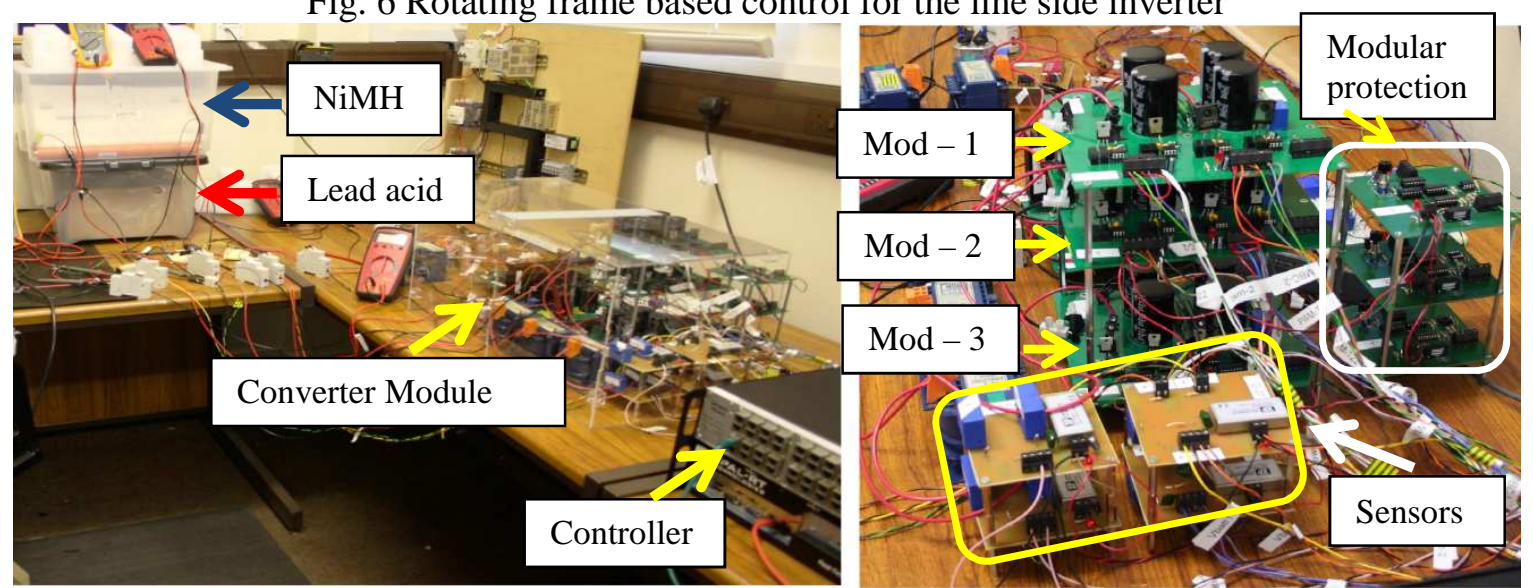

Fig. 7 Laboratory build hybrid battery energy storage system prototype
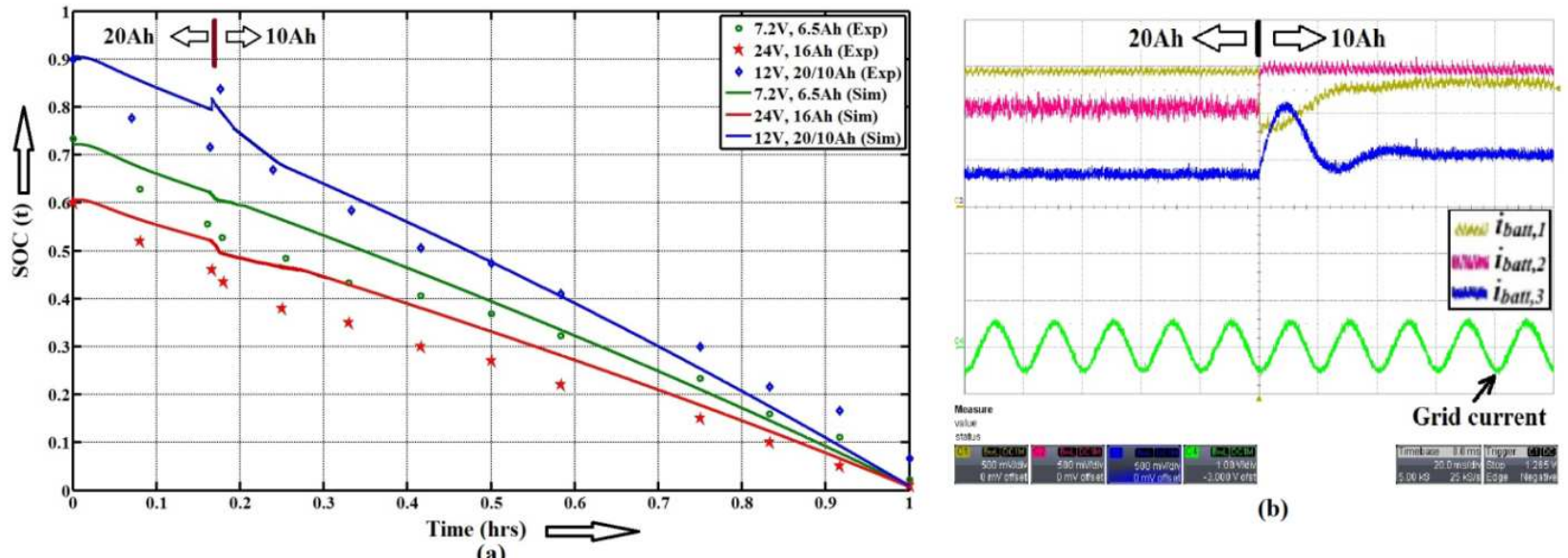

(a)

(b)

Fig. 8 Experimental result of adaptive power sharing: a) discharging trajectory under capacity fade, b) transient current sharing 


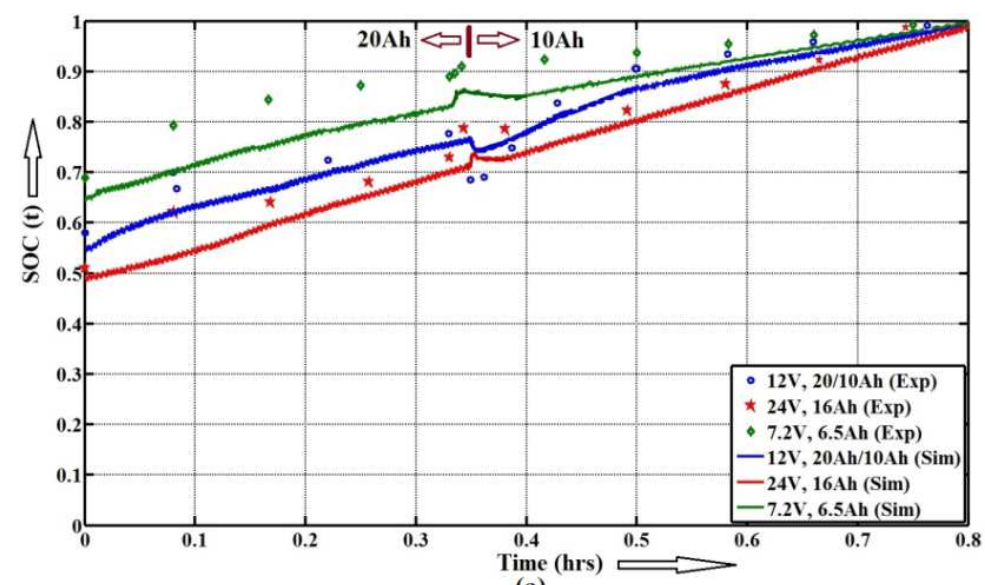

(a)

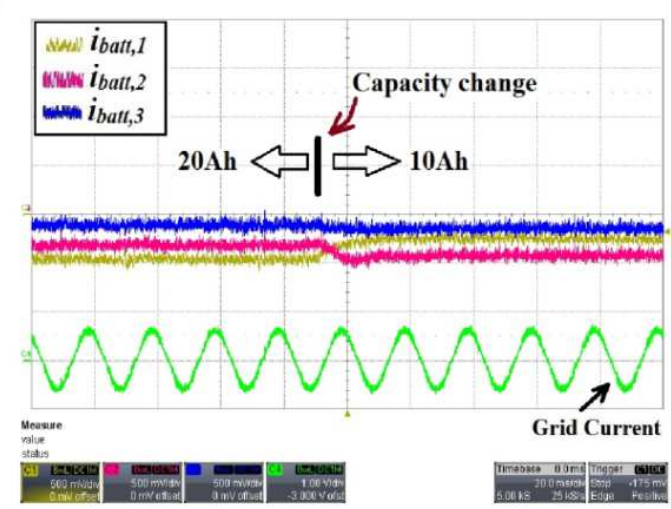

(b)

Fig. 9 Experimental result of adaptive power sharing: a) charging trajectory under capacity fade, b) transient current sharing

\section{Conclusion}

An adaptive distributed control scheme for a hybrid battery storage system under capacity fade has been proposed and experimentally validated for grid support applications. The proposed control adopts a sharing strategy to distribute the required grid power to the hybrid battery modules in an optimum manner depending on their characteristics in an adaptive nature which can detect real time capacity fade. The proposed strategy is based on an instantaneous weighting function which deals with the differences in characteristics within a set of hybrid batteries. The results show the suitability of the proposed strategy under varying parameter conditions.

\section{References}

[1] http://www.abb.com/cawp/seitp202/d3e2f486303c1d47c12577a500479955.aspx [online available].

[2] Serban, I.; Marinescu, C., "A look at the role and main topologies of battery energy storage systems for integration in autonomous microgrids," Optimization of Electrical and Electronic Equipment (OPTIM), 2010 12th International Conference on , vol., no., pp.1186,1191, 20-22 May 2010.

[3] Hao Qian; Jianhui Zhang; Jih-Sheng Lai; Wensong Yu, "A high-efficiency grid-tie battery energy storage system," Power Electronics, IEEE Transactions on , vol.26, no.3, pp.886,896, March 2011.

[4] Thomas, S.; Stieneker, M.; De Doncker, R.W., "Development of a modular high-power converter system for battery energy storage systems," Power Electronics and Applications (EPE 2011), Proceedings of the 2011-14th European Conference on, vol., no., pp.1,10, Aug. 30 2011-Sept. 12011.

[5] Hillers, A.; Biela, J., "Optimal design of the modular multilevel converter for an energy storage system based on split batteries," Power Electronics and Applications (EPE), 2013 15th European Conference on , vol., no., pp.1,11, 2-6 Sept. 2013

[6] Kawakami, N.; Ota, S.; Kon, H.; Konno, S.; Akagi, H.; Kobayashi, H.; Okada, N., "Development of a 500$\mathrm{kW}$ Modular Multilevel Cascade Convertor for battery energy storage systems," Energy Conversion Congress and Exposition (ECCE), 2013 IEEE, vol., no., pp.3375,3381, 15-19 Sept. 2013.

[7] Mukherjee, N.; Strickland, D.; "Second life Battery Energy Storage System: Converter Topology and Redundancy Selection," Power Electronics, Machines and Drives (PEMD 2014), 7th IET International Conference on , vol., no., April 2014.

[8] Einhorn, M.; Conte, F.V.; Kral, C.; Fleig, J., "A Method for Online Capacity Estimation of Lithium Ion Battery Cells Using the State of Charge and the Transferred Charge," Industry Applications, IEEE Transactions on, vol.48, no.2, pp.736,741, March-April 2012.

[9] Munoz-Condes, P.; Gomez-Parra, M.; Sancho, C.; San Andres, M.A.G.; Gonzalez-Fernandez, F.J.; Carpio, J.; Guirado, R., "On Condition Maintenance Based on the Impedance Measurement for Traction Batteries: Development and Industrial Implementation," Industrial Electronics, IEEE Transactions on , vol.60, no.7, pp.2750,2759, July 2013.

[10] Tairov, S.; Stevanatto, L.C., "Impedance measurements for battery state of health monitoring," Control, Instrumentation and Automation (ICCIA), 2011 2nd International Conference on , vol., no., pp.79,83, 2729 Dec. 2011. 